\title{
Application of Impulse Control in Smart Grid Price System Peng $\mathrm{Du}^{1,2}$, Huimin $\mathrm{Li}^{3}$ and Youping Fan ${ }^{3, "}$
}

\author{
${ }^{1}$ School of Economics and Business Administration, Chongqing University, Chongqing 404100, China \\ ${ }^{2}$ EHV Transmission Company, China Southern Power Grid, Guangzhou 510000, China \\ ${ }^{3}$ School of Electrical Engineering, Wuhan University, Wuhan 430072, China
}

Received 15 May 2013; Accepted 25 July 2013

\begin{abstract}
With the development of smart grid, flexible and interactive electricity market will finally emerge. Making full use of price can encourage consumers to use electricity during a low load period and reduce peak load, thus to guarantee electricity market stability. Impulse control is applied to price system in this paper. It is found that giving impulse control to price system can make the system reach stable state well, which is based on the theory of impulsive differential system. The sufficient condition for stable system and the max upper bound of impulse interval are also provided. The proposed method is proved to be feasible and effective by theoretically analysis and numerical simulation.
\end{abstract}

Keywords: smart grid, electricity market, price, impulse control, differential equation, stability

\section{Introduction}

Smart grid is the development direction of modern grid [1] Flexible and interactive electricity market will finally emerge. Electricity price has important meanings for healthy and robust market as the main carrier of information transmission and value distribution between the power company and consumers [2]. While price adjusting mechanism can make sure the market stable under some circumstance, it can't solve all problems. To guarantee healthy electricity market, we need government power to make full use of the price function for adjusting power supply and demand to be balanced.

In electricity price system, if there's a shortage of power supply, government can increase investment in grid construction to meet demand as possible, and this can be called an impulse phenomenon. Impulse phenomena are common in our lives. System state value would change a lot during an extremely short period when impulse phenomenon occurred. Common differential equations can't describe it, while it is available for impulsive differential equations. Nowadays, impulse control has been applied to space technology, life science, communication, information science, control system, biology and medicine. Impulsive differential equations and time-delay impulsive differential equations are used to build corresponding dynamics models to solve some problems in bio-economics [3]. Studied uncertain impulsive system robustness is researched and the application of chaotic synchronization is discussed in secret communication [4]. To solve integrated pest management problems, population ecology model with impulse is built based on impulsive differential equations theory [5]. Single

*E-mail address: ypfan@whu.edu.cn ISSN: 1791-2377 @ 2013 Kavala Institute of Technology. All rights reserved. population dynamics model is discussed with pulse birth and pulse returns [6]. Principle of comparison in impulsive differential equations is used to obtain the max interval of an advertisement [7]. Ordinary differential equations, timedelay equations, integral differential equations and impulsive differential equations are used to describe disease development and cure model after HIV infection [8].

In this paper, impulse control is applied to smart grid price system based on the analysis of impulsive differential equations applications. Taking reasonable control measures can guarantee electricity market to be stable during a long period and avoid power shortage or power surplus.

\section{Stability Principle of Impulsive Differential Equations}

Definition 1 [9] Impulse control system is described as:

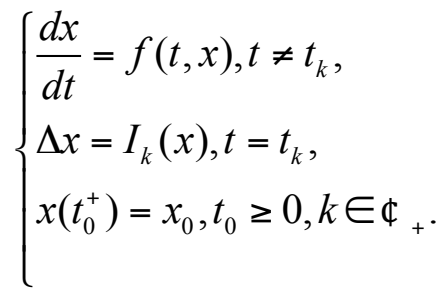

Assumed:

(H1) $0<t_{1}<t_{2}<\mathrm{L}<t_{k}<\mathrm{L}$, when $k \rightarrow \infty$, $t_{k} \rightarrow \infty$; (H2) $f:{ }^{\circ}+\times^{\circ} \rightarrow^{\circ}{ }^{n}$ is continuous on $\left(t_{k-1}, t_{k}\right] \times{ }^{\circ} n$, for each $x \in{ }^{\circ}{ }^{n}, k \in \mathbb{C}_{+}$, there exists

$$
\lim _{(t, y) \rightarrow\left(t_{k}^{+}, x\right)} f(t, y)=f\left(t_{k}^{+}, x\right) \text {. }
$$$$
\text { (H3) } I_{k}:{ }^{\circ} \rightarrow^{\circ}{ }^{n} \text {. }
$$ 
Definition 2 Assumed $V:{ }^{\circ}+\times^{\circ} \rightarrow^{\circ}$, , if:

(1) $V$ is continuous on $\left(t_{k-1}, t_{k}\right] \times^{\circ} n$, and for each $x \in{ }^{\circ}, k \in \mathbb{\Phi}_{+}$, there exists

$\lim _{(t, y) \rightarrow\left(t_{k}^{+}, x\right)} V(t, y)=V\left(t_{k}^{+}, x\right)$.

(2) $V$ satisfies Lipschitzian conditions about $x$.

Then $V \in V_{0}$.

Definition 3 Assumed $V \in V_{0}$, for each

$(t, x) \in\left(t_{k-1}, t_{k}\right] \times^{\circ}{ }^{n}$, there is:

$D^{+} V(t, x)=\lim _{h \rightarrow 0^{+}} \sup \frac{1}{h}[V(t+h, x+h f(t, x))-V(t, x)]$

Lemma 1 if:

(1) $V \in C\left({ }^{\circ}+\times S(\rho),{ }_{+}{ }_{+}\right), V \in V_{0}$,

$D^{+} V(t, x) \leq g(t, V(t, x))\left(t \neq t_{k}\right)$,

where $g \in C\left({ }^{\circ}+\times^{\circ},{ }^{\circ}\right), g(t, 0) \equiv 0$,

and $g$ satisfies condition (H2);

(2) there exists $\rho_{0}>0$, when $x \in S\left(\rho_{0}\right)$, for each $k$,

$x+I_{k}(x) \in S(\rho)$, and

$V\left(t, x+I_{k}(x)\right) \leq \psi_{k}(V(t, x))\left(t=t_{k}, x \in S\left(\rho_{0}\right)\right)$,

where $\psi_{k}:^{\circ}+\rightarrow^{\circ}$ is a monotone non-decreasing function;

(3) $b(|x|) \leq V(t, x) \leq a(|x|),(t, x) \in^{\circ}{ }_{+} \times S(\rho)$, where $a, b \in K$,

Then the impulsive differential system:

$$
\left\{\begin{array}{l}
\frac{d u}{d t}=g(t, u), t \neq t_{k} \\
u\left(t_{k}^{+}\right)=\psi_{k}\left(u\left(t_{k}\right)\right), \\
u\left(t_{0}^{+}\right)=u_{0} \geq 0
\end{array}\right.
$$

Its stability is in accordance with system (1).

Lemma 2 Assumed

$g(t, u)=\frac{d \lambda(t)}{d t} u\left(\lambda \in C^{1}\left({ }^{\circ},{ }^{\circ}{ }_{+}\right)\right)$,

$\psi_{k}(u)=d_{k} u\left(d_{k} \geq 0\right)$, based on Lemma 1 , if

$\frac{d \lambda(t)}{d t} \geq 0$, and for each $k$

$$
\lambda\left(t_{k+1}\right)+\ln d_{k} \leq \lambda\left(t_{k}\right)
$$

Then the solution $x \equiv 0$ of system (1) is stable.

\section{Mathematics Model of Electricity Price System}

Power supply is described as $S(t)$ with power demand described as $D(t)$. When power supply and power demand is balanced, the price is called equilibrium price $\bar{p}(t)$ [10]. Assumed that:

(1) $S(t)$ and $D(t)$ both are functions of real-time price $p(t)$.

(2) ideal state is $S(t)=D(t)$.

Real-time price is variable. Assumed change rate $\beta(t)$ is ratio to the difference of reserve capacity $Q(t)$ and standard reserve capacity $\bar{Q}$, that is

$$
\not(t)=\delta(\bar{Q}-Q), \delta>0
$$

Where

$$
\begin{aligned}
& Q(t)=Q_{0}+\int_{0}^{t}(S(x)-D(x)) d x \\
& S(t)=S_{0}+m p(t), S_{0}>0, m>0
\end{aligned}
$$

Power supply is nonlinear to price, so when price can be afforded, demand would stimulate price to increase, and price is positively correlated with demand. When price can't be afforded, demand would decrease as price increases, and these two are in negative correlation. So:

$$
D(t)=D_{0}-n p(t)+(\alpha p+\beta) \beta(t), D_{0}>0, n>0, \alpha>0
$$

Substitute (3), (4) and (6) to (5) and derivate $t$ :

$$
\alpha(\alpha p+\beta) \beta-\delta(m+n) p-\delta\left(Q_{0}-L_{0}\right)
$$

Turn equation (9) into first-order equations and assumed $p(t)=x(t), \beta^{\alpha}=y(t)$

$$
\left\{\begin{array}{l}
\&=y \\
\&=\delta(\alpha x+\beta) y-\delta(m+n) x-\delta\left(Q_{0}-L_{0}\right)
\end{array}\right.
$$

When $D_{0}>S_{0}$, system (10) has the only balance point $\left(\frac{D_{0}-S_{0}}{m+n}, 0\right)$, that is $\bar{p}=\bar{x}=\frac{D_{0}-S_{0}}{m+n}$.

Assumed $x_{1}(t)=x(t)-\bar{x}, x_{2}(t)=y(t)-0$, and substituted into system (10): 


$$
\left\{\begin{array}{l}
\not \&(t)=x_{2}(t) \\
\&(t)=\delta\left(\alpha x_{1}+\beta^{\prime}\right) x_{2}-\delta(m+n) x_{1}
\end{array}\right.
$$

Where $\beta^{\prime}=\alpha \bar{x}+\beta$. Thus, balance point stability analysis of system (10) is turned into zero solution stability analysis of system (11).

\section{Impulse Control In Electricity Price System}

According to [11]: when $\beta^{\prime} \geq 0$, electricity price is far away from equilibrium price, and electricity market is unstable. Thus we put into government control as impulse control at discrete time, making balance point become stable to guarantee healthy market. For easy study, we divided system (11) into linear part and nonlinear part, that is

$$
\&=A x+g(x)
$$

Where

$$
x^{T}=\left(x_{1}, x_{2}\right), A=\left(\begin{array}{cc}
0 & 1 \\
-\delta(m+n) & 0
\end{array}\right), g(x)=\left(\begin{array}{c}
0 \\
\delta\left(\alpha x_{1}+\beta^{\prime}\right) x_{2}
\end{array}\right)
$$

Assumed control impulse $I_{k}=B x, B=\left(\begin{array}{cc}B_{1} & 0 \\ 0 & B_{2}\end{array}\right)$. Thus, we got the impulse control system:

$$
\left\{\begin{array}{c}
\mathbb{R}=A x+g(x), t \neq t_{k} \\
\Delta x=B x, t=t_{k}
\end{array}\right.
$$

The aim of impulse control is to seek for control parameters to make system (13) stable.

\section{Stability Analysis}

Theorem 1 Assumed $\xi_{\max }$ is the max-eigenvalue of matrix $A+A^{T}, d_{k}=\max \left\{\left(1+B_{1}\right)^{2},\left(1+B_{2}\right)^{2}\right\}$, $\xi=\xi_{\max }+2 \delta\left(\alpha M_{x}+\beta^{\prime}\right)$, where $M_{x}=\max \left\{x_{1}, x_{2}\right\}$. If the following condition is satisfied:

$$
0 \leq \xi\left(t_{k+1}-t_{k}\right) \leq-\ln d_{k}
$$

Then system (13) is stable.

Prove: structure a Lyapunov function,

$$
V=x^{T} x
$$

When $t \neq t_{k}$,

$$
D^{+} V(t, x)=x^{T}\left(A^{T}+A\right) x+g^{T}(x) x+x^{T} g(x)(16)
$$

Where,

$$
\begin{aligned}
x^{T}\left(A^{T}+A\right) x \leq & \xi_{\max } V(t, x) \\
g^{T}(x) x+x^{T} g(x) & =2 \delta\left(\alpha x_{1}+\beta^{\prime}\right) x_{2}^{2} \\
& =2\left[x_{1}(t) x^{T}\left(\begin{array}{cc}
0 & 0 \\
0 & \delta \alpha
\end{array}\right) x+x^{T}\left(\begin{array}{cc}
0 & 0 \\
0 & \delta \beta^{\prime}
\end{array}\right) x\right] \\
& \leq 2 \delta\left(\alpha M_{x}+\beta^{\prime}\right) V(t, x)
\end{aligned}
$$

So,

$$
D^{+} V(t, x) \leq \xi V(t, x)
$$

Assumed $g(t, u)=\xi u$, so condition (1) of Lemma 1 is satisfied.

$$
\forall \rho>0, x \in S(\rho):
$$

$$
\|x+B x\| \leq\|E+B\|\|x\| \leq\|x\|
$$

So, $x+B x \in S(\rho)$.

When $t=t_{k}$ :

$$
V(t, x+B x)=x^{T}(E+B)^{T}(E+B) x \leq d_{k} V
$$

Assumed $\psi_{k}(u)=d_{k} u$, so condition (2) of Lemma 1 is satisfied.

For $V=x^{T} x=\|x\|^{2}$, there must exist $a, b \in K$ to make the following in-equations:

$$
b(\|x\|) \leq V(t, x) \leq a(\|x\|)
$$

So condition (3) of Lemma 1 is satisfied.

According to Lemma 1, stability of system (13) is in accordance with the following system:

$$
\left\{\begin{array}{l}
\frac{d u}{d t}=g(t, u), t \neq t_{k} \\
u\left(t_{k}^{+}\right)=\psi_{k}\left(u\left(t_{k}\right)\right) \\
u\left(t_{0}^{+}\right)=u_{0} \geq 0
\end{array}\right.
$$

$$
\begin{array}{r}
\text { When } 0 \leq \xi\left(t_{k+1}-t_{k}\right) \leq-\ln d_{k} \text { : } \\
\xi t_{k+1}+\ln d_{k} \leq \xi t_{k}
\end{array}
$$

As $g(t, u)=\xi u$, we assumed $\lambda(t)=\xi t$, then $\lambda\left(t_{k+1}\right)+\ln d_{k} \leq \lambda\left(t_{k}\right)$ is satisfied. 
According to Lemma 2, system (13) is stable.

\section{Numerical Simulation}

We simulated the ideal electricity price system (10) with data of N.Y.C in July, 2012 from NYISO.

The parameters are set as following:

$\delta=0.8, m=20 M W H^{2} / \$, n=20 M W H^{2} / \$$, $\alpha=0.8 \quad, \quad \beta=-12 \quad, \quad S_{0}=8230 M W H$, $D_{0}=9658 M W H, M_{x}=8$.

Thus:

$$
\bar{p}=\frac{L_{0}-Q_{0}}{m+n}=\frac{9658-8230}{20+20}=35.7 \$ / M W H
$$

When there is no impulse control put into system (10), the system is unstable. After impulse control put into system (10), the system figure is as Fig.1 shows. Assumed initial value $x(0)=(0.75,1.25)^{T}$.

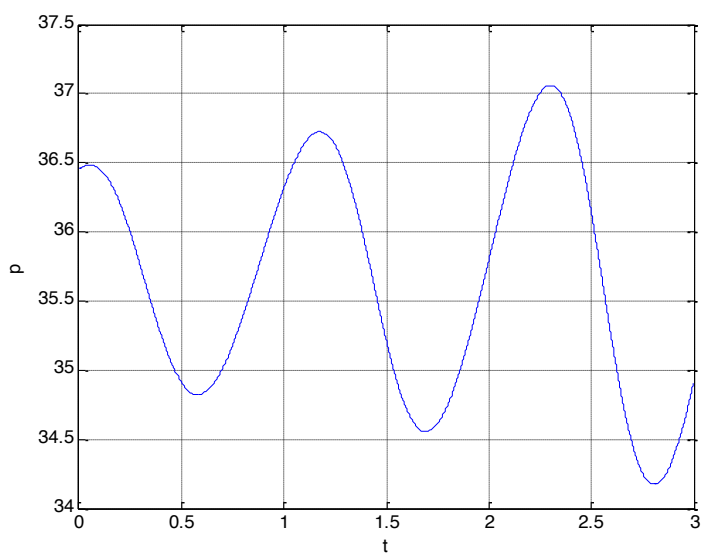

(a)

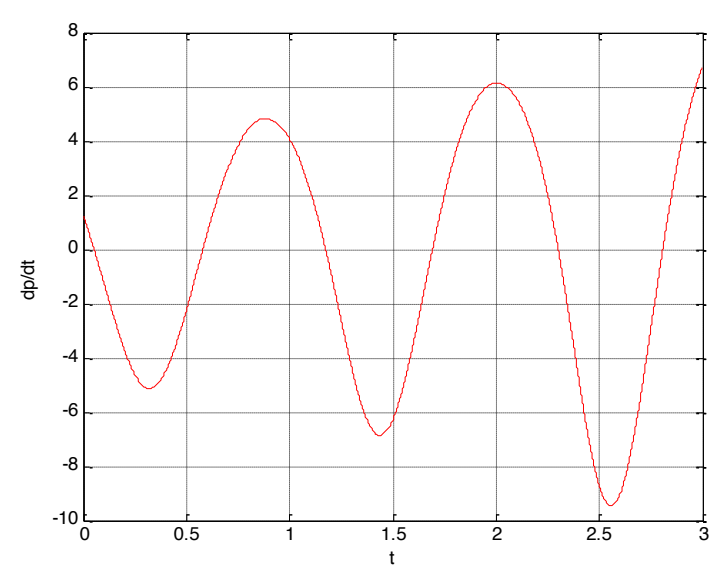

(b)

Fig.1 Price System Without Impulse Control
Set $B=\left(\begin{array}{cc}-0.95 & 0 \\ 0 & -0.96\end{array}\right)$
then $d_{k}=\max \left\{\left(1+B_{1}\right)^{2},\left(1+B_{2}\right)^{2}\right\}=0.0025$

As Fig. 2 shows, the system becomes stable.

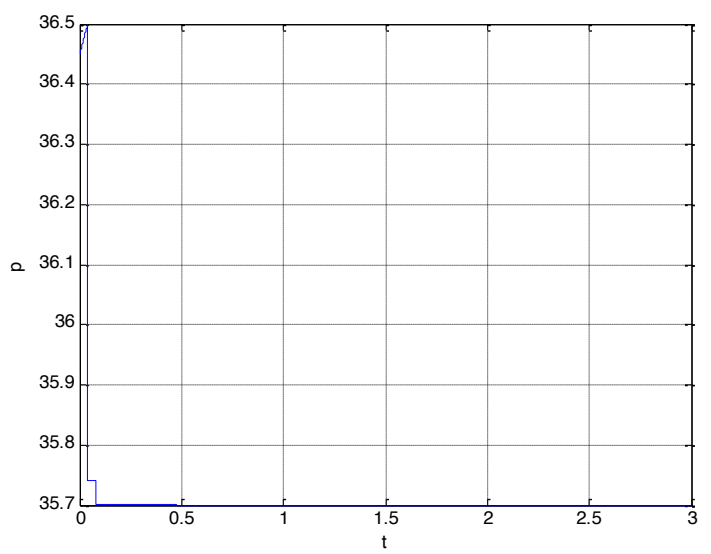

(a)

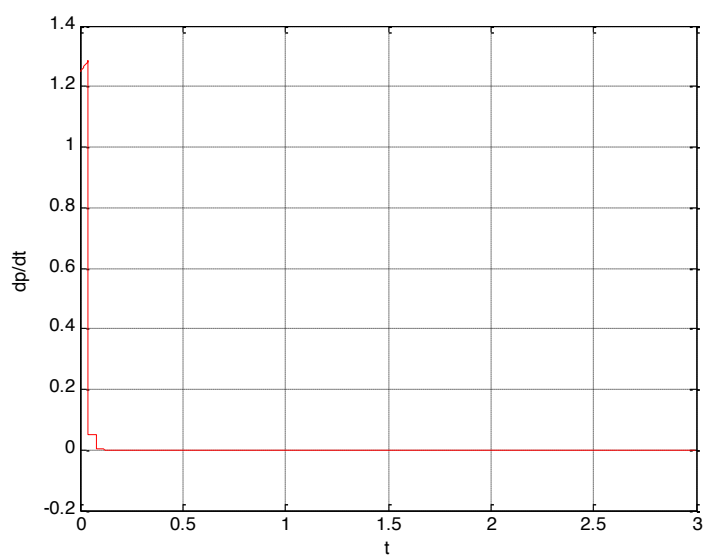

(b)

Fig.2 Price System With Impulse Control

From the above MATLAB simulation, we can see that when there is no impulse control put into the ideal electricity price system (10), price and change rate both have rectilinear oscillations. But after the government makes some adjusting measures, the system becomes stable and the market becomes balanced.

\section{Conclusion}

Price adjustment mechanism exists objectively in electricity market and it works under certain conditions. But this kind of mechanism is limited. To guarantee the healthy development of electricity market, we need government to help control the market. When power supply is in short or in surplus, government can use economic means to adjust electricity price, even some administrative means are allowed at some serious period. In this paper, we used comparison principle of impulsive differential equations to study impulse control of electricity price system. Making balance point stable with impulse control, we provided 
sufficient condition for controlling price model stable and the upper bound estimation of impulse control interval. The method is proved to be effective by simulating system in Matlab and the results are good. Therefore, this method can guide the future smart grid developing stably, healthily and continuously. Theory basis is also provided for decisionmaking organization, and it can help them to take effective measures to make sure electricity market reach a stable state fast.

\section{References}

1. Zhaoli Feng, Jiajia Mao, Shusheng Wen, Tianmeng Liang, "Review on Real-time Pricing for Smart Grid: Models and Optimization Methods", Industrial Control Computer 25(2), pp. 87-90, 2012.

2. Yong Zeng, "Study on the Real-time Electricity Price Based on the Smart Grid", Chongqing University, Chongqing, 2011.

3. Jianjun Jiao, "Application of Impulsive Differential Equations In Bio-economics", Dalian University of Technology, Dalian, 2008.

4. Guangren Duan, "Impulsive Control Theory and Application", Harbin Institute of Technology, Harbin, 2007.

5. Dongjuan Yue, "Application of Impulsive Differential Equations In Agricultural Pest Management", South Central University, Changsha, 2011.
6. Lei Bao, Jianjun Jiao, "One Population Dynamics Model With Impulse Effect", Mathematics In Practice and Theory, 41(5), pp.162-166, 2011.

7. Dilan Chen, Jitao Sun, "Impulsive Control of An Advertising System”, Journal of Biomathematics, 20(1), pp.17-22, 2005.

8. Yan Wang, Yicang Zhou, "Mathematical Modeling and Dynamics of HIV Progression and Treatment", Chinese Journal of Engineering Mathematics, 27(3), pp.534-548, 2010.

9. Xinyu Song, Hongiian Guo, Xiangyun Shi, Impulsive Differential Equations Theory and Its Application, Science Press: Beijing, 2011.

10. Jiejie Cui, "Stability Analysis and Control of Economic System With Time-delay Impulse", Jiangsu University, Zhenjiang, 2009.

11. Shuhe Wang, Differential Equation Models and Chaos, University of Science and Technology of China Press: Hefei, 1999. 